Boletín de la Sociedad Geológica Mexicana

VOLUMEN 62, NÚM. 1, 2010, P. 43-53

\title{
Estudio comparativo de la peligrosidad de jales en dos zonas mineras localizadas en el sur y centro de México
}

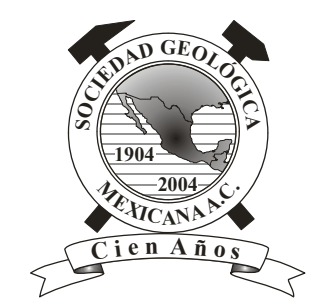

\author{
Francisco Martín Romero ${ }^{*}$, Margarita Gutiérrez Ruíz ${ }^{2}$ \\ ${ }^{1}$ Instituto de Geología, Universidad Nacional Autónoma de México. Ciudad Universitaria, 04510 México, D.F. \\ ${ }^{2}$ Instituto de Geografía, Universidad Nacional Autónoma de México. Ciudad Universitaria, 04510 México, D.F. \\ *fmrch@geologia.unam.mx
}

\begin{abstract}
Resumen
Se realizó un estudio geoquímico y mineralógico en jales de dos zonas mineras localizadas en el sur y centro de México. Consecuente con la composición mineralógica identificada en los jales estudiados, éstos se caracterizan por contener elementos potencialmente tóxicos como arsénico (140 - 3627 mgkg-1 $)$, cadmio $\left(0.5-434 \mathrm{mgkg}^{-1}\right)$, plomo (148 - $\left.10900 \mathrm{mg} \mathrm{kg}^{-1}\right)$, cobre $(0.002-1.55 \%)$, zinc $(0.021-3.86$ \%) y hierro (2.4 - 35.7\%). Sin embargo, las concentraciones totales de los otros elementos potencialmente tóxicos (EPT) como bario, cromo, mercurio, plata, plomo, selenio, berilio, talio, níquel y vanadio son bajas e inferiores a las concentraciones totales que podrían representar un riesgo para el ambiente y la salud de acuerdo a la Norma Oficial Mexicana de suelos contaminados NOM-147.

En los dos sitios de estudio se identificaron jales oxidados de color café y jales inalterados de color gris. En el $60 \%$ de las muestras de jales oxidados del sur de México se observaron valores de pH que varían de 2.4 a 3.7, lo que indica la formación de drenaje ácido producto de la intensa oxidación de los sulfuros metálicos. Sin embargo, en el $40 \%$ de las muestras de estos jales oxidados el $\mathrm{pH}$ es prácticamente neutro o ligeramente básico (6.5-8.2), lo que indica que la acidez generada por la oxidación de los sulfuros fue consumida por minerales con capacidad de neutralización. De la misma manera, los valores de $\mathrm{pH}$ de los jales oxidados del centro de México, varían de 6.5 a 8.2 , observándose en este caso todavía la presencia de calcita y wollastonita (basicidad residual).

Los resultados de las pruebas de balance ácido-base permiten pronosticar que los jales inalterados y grises del sur de México serán generadores de acidez cuando se oxiden, debido a que tienen un alto potencial de acidez "PA" (media $=206 \mathrm{~kg} \mathrm{CaCO}_{3} / \mathrm{t}$ ) y bajo potencial de neutralización "PN" (media $=121 \mathrm{~kg} \mathrm{CaCO}_{3} /$ ton), lo que indica que no tienen suficiente cantidad de minerales neutralizadores para consumir la acidez generada. La relación de PN / PA de estos jales (media $=0.6$ ) indica que son generadores potenciales de drenaje ácido.

Las Regulaciones Ambientales Mexicanas señalan que los jales que tienen una relación de PN/PA menor a 1.2 deben de ser clasificados como generadores potenciales de drenaje ácido. Sin embargo, los jales inalterados del centro de México se pueden clasificar como no generadores potenciales de acidez debido a que su "PN" (media $=414 \mathrm{~kg} \mathrm{CaCO}_{3} / \mathrm{t}$ ) es más alto que su "PA" (media $=71 \mathrm{~kg} \mathrm{CaCO}_{3}$ /t) con un valor promedio de la relación PN / PA igual 5.8, lo que indica que la acidez que se pueda generar, por la oxidación de los sulfuros metálicos, será eficientemente neutralizada.

Las mayores concentraciones de EPT disueltos se encontraron en los lixiviados ácidos del sur de México (arsénico $=0.4 \mathrm{mg} \mathrm{L}^{-1}$, cadmio $=1.3 \mathrm{mg} \mathrm{L}^{-1}$, plomo $=0.2 \mathrm{mgL}^{-1}$, zinc $=55 \mathrm{mg} \mathrm{L}^{-1}, \mathrm{y}$ hierro $\left.=28 \mathrm{mg} \mathrm{L}-1\right)$, mientras que en los lixiviados con pH cercano al neutro de los dos sitios de estudio se encontraron bajas concentraciones de EPT disueltos, lo que indica su baja movilidad. La baja movilidad de los EPT en los jales inalterados grises se puede explicar debido a que éstos están asociados a los sulfuros metálicos, que son minerales estables bajo las condiciones actuales. En los jales oxidados, la baja movilidad de los EPT está relacionada con procesos de sorción en superficies de oxihidróxidos de Fe.
\end{abstract}

Palabras clave: drenaje ácido, arsénico, metales pesados, México. 
Tailings from two mining zones located in the south and centre of México were geochemically and mineralogicaly studied. According to the identified mineralogical composition, these studied tailings are characterized by its content of arsenic $\left(140-3627 \mathrm{mg} \mathrm{kg}^{-1}\right), \mathrm{cadmium}^{-}$ (0.5-434 $\left.\mathrm{mg} \mathrm{kg}^{-1}\right)$, lead (148-10900 $\left.\mathrm{mg} \mathrm{kg}^{-1}\right)$, copper (0.002-1.55\%), zinc (0.021-3.86\%) and iron (2.4-35.7\%). Nevertheless, the total concentrations of the other potentially toxic elements (PTE) like barium, chromium, mercury, silver, lead, selenium, beryllium, thallium, nickel and vanadium are below the concentrations limits that represent an environmental and health potential risk established by Mexican Environmental Regulations of contaminated soils NOM-147.

In both sites oxidized tailings (brown color) and reduced tailings (gray color) were identified. $60 \%$ of the oxidized samples from the south of Mexico presented acid pH values from 2.4 to 3.7, indicating the formation of acidic drainage as a consequence of extensive sulfide minerals oxidation. However, the other $40 \%$ of these oxidized samples showed practically neutral or slightly basic pH values (6.5-8.2) as a consequence of the action of neutralizing minerals. In the same way, the pH values found in the oxidized tailings from central Mexico varied between 6.5 and 8.2, being still possible to find calcite and wollastonite (residual alkalinity) in these cases.

Acid-base accounting results allow predicting that unoxidized gray tailings from south Mexico will be acid generating, when they

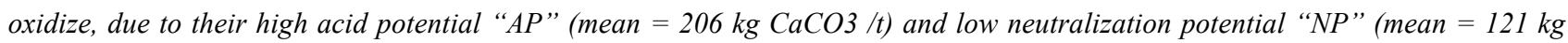
$\mathrm{CaCO} 3$ /ton), which indicate insufficiency of $\mathrm{pH}$-buffering minerals to consume acid generation. The ratio PN/PA of these tailings (mean $N P / A P=0.6$ ) indicates that they are generating potentials of acid drainage. The Mexican environmental regulations indicate that tailings with a ratio of NP/AP less than 1.2 must be classified as potential generators of acid drainage

However, unoxidized gray tailings from centre Mexico may be classified as non-acid forming because it's "NP" (mean = $414 \mathrm{~kg}$ $\mathrm{CaCO} 3 / \mathrm{t}$ ) is higher than its "AP" (mean $=71 \mathrm{~kg} \mathrm{CaCO3/t)}$ with a ratio of $\mathrm{NP} / \mathrm{AP}$ of 5.8 , and therefore sufficient pH-buffering minerals are present to balanced acid generation.

The highest concentrations of dissolved PTE were found in the acidic tailings from south Mexico (arsenic $=0.4 \mathrm{mg} \mathrm{L}^{-1}$, cadmium

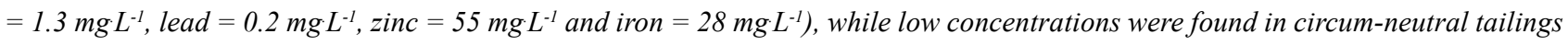
from both studied sites, showing low mobility of these PTE. The low mobility of PTE in unoxidized grays tailings may be explained because they are forming sulfide minerals which are stable under environmental conditions. In oxidized tailings, the low mobility of the EPT is related to sorption processes onto Fe oxy-hydroxides.

Key words: acid drainage, arsenic, heavy metals, Mexico

\section{Introducción}

México es un país con abundantes recursos minerales, lo que ha marcado su tradición minera. Entre los principales tipos de yacimientos que se han explotado destacan los epitermales (Taxco, Guanajuato, Pachuca, Zacatecas, Santa Bárbara, etc.), los de metasomatismo de contacto o skarn (Charcas, Zimapán, La Paz, San Martín, etc.), los sulfuros masivos vulcanogenéticos (Tizapa, Rey de Plata, etc), y yacimientos del tipo pórfido cuprífero (La Caridad, Cananea, etc). En estos diferentes tipos de yacimientos ocurren tanto los minerales económicos como los no económicos, y con el fin de separarlos son sometidos a diferentes procesos físicos y químicos. El avance de la tecnología en la industria minera mundial propició la introducción en México de procesos metalúrgicos como la flotación y la cianuración a inicios del siglo $\mathrm{XX}$, lo que ha permitido explotar mayores volúmenes de mineral con valor comercial, a la vez que propició la generación de mayor cantidad de residuos, entre los que destacan los denominados "jales".

Los jales que se generan en el proceso de concentración de minerales de plomo, plata, zinc y cobre, generalmente contienen sulfuros metálicos residuales como la pirita $\left(\mathrm{FeS}_{2}\right)$, pirrotita $\left(\mathrm{Fe}_{1-\mathrm{x}} \mathrm{S}\right)$, galena $(\mathrm{PbS})$, esfalerita $(\mathrm{ZnS})$, calcopirita $\left(\mathrm{CuFeS}_{2}\right)$ y arsenopirita (FeAsS) que son la fuente de elementos potencialmente tóxicos (EPT) como arsénico $(\mathrm{As})$, cadmio $(\mathrm{Cd})$, plomo $(\mathrm{Pb})$, cobre $(\mathrm{Cu})$, zinc $(\mathrm{Zn})$, hierro (Fe), etc. (Romero et al., 2007). El principal problema ambiental asociado a los jales está relacionado con la generación de drenaje ácido y su dispersión a través de los escurrimientos superficiales (dispersión hídrica). El drenaje ácido se genera por la oxidación de los sulfuros metálicos y forma soluciones que se caracterizan por tener valores bajos de $\mathrm{pH}$ y altas concentraciones de EPT disueltos (Lin, 1997; Johnson et al., 2000; Moncur et al., 2005) que al transportarse, pueden convertirse en un problema ambiental severo al contaminar suelos, sedimentos, aguas superficiales y aguas subterráneas (Bain et al., 2000; Armienta et al., 2001; Jung, 2001).

Para que ocurra la oxidación en los jales, es necesario que contengan sulfuros metálicos reactivos y que existan las condiciones climáticas apropiadas (aire y agua o atmósfera húmeda). La oxidación de los sulfuros metálicos en los jales es, generalmente, muy limitada durante la operación de la mina y se desarrolla lentamente a lo largo del 
tiempo, después que cesa la acumulación en el depósito y la porosidad en el mismo permite la difusión del oxígeno atmósferico. Antes de que ocurra la oxidación de los jales, éstos no presentan signos visibles de alteración y por lo general son de color gris. Cuando ocurre la oxidación de los sulfuros metálicos, los jales presentan una coloración café, amarilla o roja.

Es importante destacar que la oxidación de los sulfuros metálicos no siempre produce drenaje ácido, ya que la tendencia de los jales para generarlo es una función del balance entre los minerales productores de ácido (sulfuros metálicos) y los minerales con capacidad de neutralización (carbonatos, hidróxidos y aluminosilicatos). En general, cuando la capacidad de neutralización excede la capacidad de generación de drenaje ácido, se consumirá toda la acidez generada y las soluciones que drenen del depósito de jales tendrán un $\mathrm{pH}$ cercano al neutro.

Actualmente no existe una estimación confiable de la cantidad de depósitos de jales distribuidos en el territorio de la República Mexicana. Ramírez (2001), estima que en México existen poco más de 80 almacenamientos de jales en operación. Sin embargo, no existe un inventario de la cantidad y situación de las presas de jales inactivas abandonadas. Históricamente, los jales generados fueron depositados en los alrededores de las minas en sitios topográficamente bajos sin tomar en cuenta alguna medida de protección ambiental, convirtiéndose en focos de contaminación potencial para la región donde están ubicados.

Se seleccionaron los depósitos de jales de dos Unidades Mineras localizadas en el sur y centro de México (Figura 1) para realizar un estudio geoquímico y mineralógico con los objetivos de:

1) Evaluar la peligrosidad de los jales en función de:

1.1) La concentración total de elementos potencialmente tóxicos (EPT).

1.2) Capacidad de generación de drenaje ácido.

1.3) Concentración geodisponible de EPT.

2) Identificar los minerales primarios y secundarios en los jales y establecer la relación con la peligrosidad.

3) Identificar los principales procesos geoquímicos que gobiernan la movilidad de los EPT.

\section{Contexto geográfico y geológico}

En la Unidad Minera ubicada en el sur de México predomina un clima tropical subhúmedo y la temperatura promedio anual es de $28^{\circ} \mathrm{C}$. El registro histórico de precipitaciones indica un promedio anual del orden de los $1000 \mathrm{~mm}$. La precipitación máxima ocurre en el mes de septiembre con valores de $300 \mathrm{~mm}$ y la precipitación mínima ocurre en el mes de febrero con valores de $1.2 \mathrm{~mm}$ (INEGI, 1999).

Las rocas que afloran en esta zona conforman una secuencia litológica que está representada, de la base a la cima, por rocas metamórficas, sedimentarias e ígneas
(IMMSA, 1973). Las rocas más antiguas son esquistos con intercalaciones de pizarras del Paleozoico Superior. Sobre estos esquistos yacen discordantemente rocas sedimentarias (calizas, dolomitas, lutitas calcáreas, areniscas y conglomerados) de las Formaciones Morelos y Mexcala del Cretácico Inferior y Superior. Rocas clásticas de origen continental de la Formación Balsas del Terciario yacen sobre las rocas sedimentarias del Cretácico, y finalizando la columna litológica se encuentran las rocas riolíticas de la Formación Tilzapotla, que junto a algunas intrusiones ígneas de tipo basáltico son las rocas más jóvenes de la región. Los yacimientos minerales importantes en esta Unidad Minera son vetas epitermales con sulfuros de $\mathrm{Pb}$ y $\mathrm{Zn}$ encajonadas en lutitas calcáreas, calizas y areniscas del Cretácico. Los sulfuros metálicos reportados son pirita $\left(\mathrm{FeS}_{2}\right)$, esfalerita $(\mathrm{ZnS})$, galena $(\mathrm{PbS})$ y arsenopirita (FeAsS). Los minerales transparentes más abundantes son cuarzo, calcita y feldespatos potásicos (IMMSA, 1973).

En la Unidad Minera localizada en la parte central de México, predomina un clima seco templado, semiseco templado y seco semicálido y hay una temperatura promedio anual de $17^{\circ} \mathrm{C}$. El registro histórico de precipitaciones indica un promedio anual del orden de los $391 \mathrm{~mm}$. La precipitación máxima ocurre en el mes de julio con valores de $93 \mathrm{~mm}$ y la precipitación mínima ocurre en el mes de febrero con valores de $10.2 \mathrm{~mm}$. En la zona se reportan valores de evaporación potencial anual de 1942 mm (CNA, 2002).

La secuencias litológica de la zona está conformada por rocas calizas de la Formación Zuloaga (Jurásico) que se divide en dos unidades, la primera de ellas se compone de capas de $1 \mathrm{~m}$ de caliza densa de aproximadamente 1 $\mathrm{m}$ de espesor, alternando con capas de 3 a $4 \mathrm{~m}$ de caliza microcristalina. Sobre las calizas de la Formación Zuloaga yacen las calizas de la Formación Cuesta del Cura (Cretácico), que se compone de estratos delgados a medios con intercalaciones de horizontes arcillosos y abundantes bandas de pedernal negro. Las calizas de ambas Formaciones están afectadas por rocas intrusivas ácidas del Terciario. La secuencia litológica finaliza con los materiales aluviales del Cuaternario (Romero, 2007a). Los depósitos minerales que se han extraído y beneficiado se identifican como cuerpos de reemplazamiento (skarn) y relleno de fisuras (vetas). Los cuerpos de reemplazamiento se encuentran encajonados en calizas del Jurásico-Cretácico y están asociados a zonas de contacto con los intrusivos ácidos. La mineralización característica del relleno de fisura (de menor potencialidad) está asociada a vetas encajonadas en el contacto entre las calizas y los intrusivos. Los sulfuros metálicos reportados son: pirita $\left(\mathrm{FeS}_{2}\right)$, esfalerita $(\mathrm{ZnS})$, galena $(\mathrm{PbS})$, calcopirita $\left(\mathrm{FeCuS}_{2}\right)$, argentita $\left(\mathrm{Ag}_{2} \mathrm{~S}\right)$ y arsenopirita (FeAsS). Entre los minerales de ganga se reportan calcita $\left(\mathrm{CaCO}_{3}\right)$, cuarzo $\left(\mathrm{SiO}_{2}\right)$, wollastonita $\left(\mathrm{CaSiO}_{3}\right)$, grosularita $\left(\mathrm{Ca}_{3} \mathrm{Al}_{2}\left(\mathrm{SiO}_{4}\right)_{3}\right)$ y damburita $\left(\mathrm{CaB}_{2}\left(\mathrm{SiO}_{4}\right)_{2}\right.$, (Romero, 2007a). 

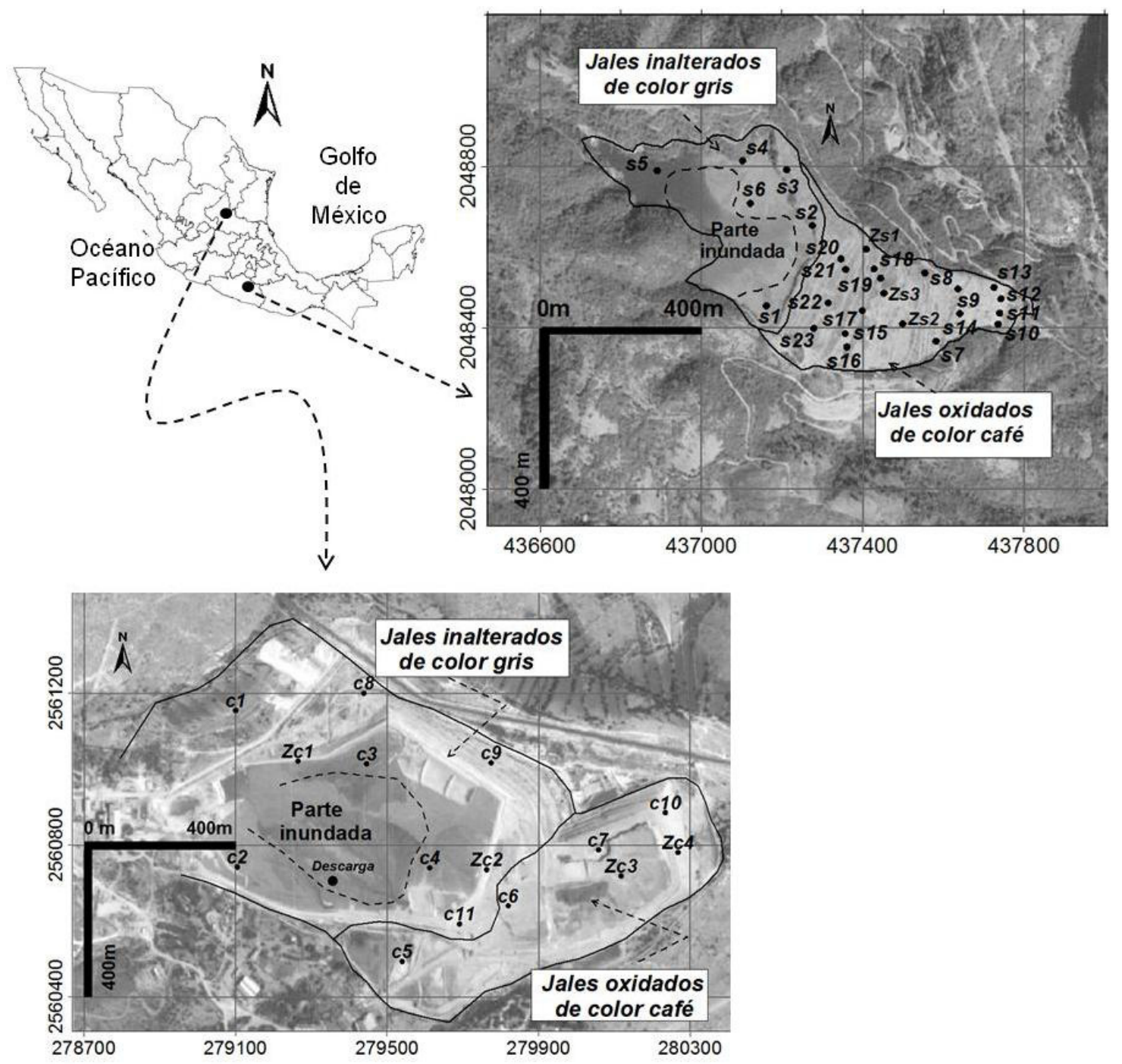

Figura 1 Ubicación de los depósitos de jales estudiados: (a) Muestras de jales de la Unidad Minera del sur de México: $\mathrm{s} 1$ - s23 corresponden a las muestras superficiales (total $=23$ muestras); Zs1 - Zs3 corresponde a los perfiles de 130 a $300 \mathrm{~cm}$ de profundidad donde se tomaron 14 muestras. (b) Muestras de jales de la Unidad Minera del centro de México: c1 - c11 y descarga corresponden a muestras superficiales (total = 12 muestras); Zc1 - Zc4 corresponde a los perfiles de $100 \mathrm{~cm}$ de profundidad donde se tomaron 16 muestras.

\section{Metodología}

En los jales de la Unidad Minera localizada en el sur de México se colectaron 37 muestras de las cuales 23 son superficiales y 14 se tomaron en perfiles de hasta $300 \mathrm{~cm}$ de profundidad. En los jales de la Unidad Minera localizada en el centro de México se colectaron 28 muestras de las cuales 12 son superficiales y las otras 16 se tomaron hasta la profundidad de $100 \mathrm{~cm}$. Las muestras de jales de los dos sitios corresponden tanto a jales de color gris que no presentan signos visibles de alteración, como a jales oxidados de color café (Figura 1). Para valorar la peligrosidad de los jales de estudio, en todas las muestras se determinaron las concentraciones totales de los elementos potencialmente tóxicos (EPT) regulados en la Norma Oficial Mexicana de jales NOM-141 (SEMARNAT, 2004): arsénico, bario, cadmio, cromo, mercurio, plata, plomo, selenio. También se determinaron las concentraciones totales de otros elementos potencialmente tóxicos como berilio, talio, níquel y vanadio, los cuales están regulados en la Norma Oficial Mexicana de suelos contaminados NOM-147 (SEMARNAT, 2007). Adicionalmente se determinaron las concentraciones totales de otros EPT como el cobre, hierro y zinc, que son elementos propios de los yacimientos minerales de los que provienen los residuos estudiados.

Para determinar la concentración total de los EPT, las muestras se pulverizaron hasta obtener una granulometría inferior a la malla 200 y fueron digeridas con ácido nítrico concentrado en horno de microondas siguiendo el procedimiento general descrito en el método 3051 de la US-EPA (1994). También se determinaron las concentraciones geodisponibles de los EPT de interés en todas las muestras 
de jales, de acuerdo al procedimiento establecido en la Norma Oficial Mexicana NOM-141 (SEMARNAT, 2004), que consiste en obtener extractos para análisis químicos a partir de la agitación de las muestras sólidas con agua en equilibrio con la atmósfera $(\mathrm{pH}=5.5 \pm 0.2)$ en una relación sólido:líquido de 1:20. Estos extractos representan los lixiviados que se formarían en los jales al contacto con agua de lluvia.

Los análisis de las concentraciones totales y geodisponibles de los EPT de interés se realizaron por Espectroscopia de Emisión Atómica Inductivamente Acoplada a Plasma (ICP-AES). La calidad de los resultados de los análisis químicos se controló a través de análisis de muestras duplicadas (precisión del método) y análisis del material de referencia para jales mineros RTS-3 "Canadian Certified Reference Materials" (exactitud del método). Los análisis de las muestras duplicadas indicaron que el error de la reproducibilidad varía entre 3 y $8 \%$, mientras que el porcentaje de error entre las concentraciones reportadas del material de referencia y las determinadas en este estudio varía entre 8 y $10 \%$.

La capacidad de generación de ácido en los jales oxidados -donde ya ocurrieron los procesos de oxidación y neutralización- se determinó mediante la medición del $\mathrm{pH}$ en los extractos acuosos, para lo cual se prepararon suspensiones de las muestras sólidas homogenizadas en una relación sólido:agua de 1:5, siguiendo el procedimiento general descrito en el método 9045 (US-EPA, 1995). En el caso de los jales grises que no presentan signos visibles de alteración, para pronosticar la capacidad de generación de drenaje se realizaron pruebas de balance ácido - base (BAB), utilizando el método "Prueba Modificada de Balance Ácido-Base" (Lawrence y Wang, 1997), que es el señalado en la Norma Oficial Mexicana NOM-141 (SEMARNAT, 2004). Esta prueba de BAB consiste en determinar en la muestras de jales: i) El Potencial de neutralización (PN), que está dado por la presencia de minerales que contienen carbonatos, principalmente calcita. ii) El Potencial de acidez (PA), que está dado por la presencia de minerales que contienen azufre, principalmente pirita.

La identificación de las fases sólidas en 10 muestras seleccionadas de jales de cada sitio de estudio se realizó utilizando la técnica de difracción de rayos X (DRX) y microscopía electrónica de barrido acoplada con espectrometría de Rayos X por energía dispersa (MEB-EDS). Considerando que los elementos potencialmente tóxicos están, principalmente, asociados a las partículas más finas (Roussel et al., 2000), los análisis para la identificación de las fases sólidas en los jales de estudio, se realizaron en las muestras una vez que éstas fueron tamizadas con el fin de concentrar las partículas inferiores a los $38 \mu \mathrm{m}$.

\section{Resultados y Discusión}

\subsection{Mineralogía}

Los resultados del análisis mineralógico se muestran en la Tabla 1. En la composición mineralógica de las muestras de jales grises e inalterados de las dos Unidades Mineras predominan los minerales primarios como cuarzo $\left(\mathrm{SiO}_{2}\right)$, calcita $\left(\mathrm{CaCO}_{3}\right)$, feldespatos $\left(\mathrm{KAlSi}_{3} \mathrm{O}_{8}\right)$, pirita $\left(\mathrm{FeS}_{2}\right)$, esfalerita $(\mathrm{ZnS})$ y galena $(\mathrm{PbS})$. También se identificaron oxihidróxidos de hierro formando aureolas alrededor de la pirita, cuya presencia puede interpretarse como el producto de alteración de los sulfuros de Fe en los yacimientos de los cuales provienen los jales estudiados. Adicionalmente, en los jales grises e inalterados del centro de México se identificaron calcopirita $\left(\mathrm{FeCuS}_{2}\right)$ y wollastonita $\left(\mathrm{CaSiO}_{3}\right)$.

Tabla1 Composición mineralógica de los jales de estudio

\begin{tabular}{|c|c|c|c|c|}
\hline \multirow{2}{*}{ Minerales } & \multicolumn{2}{|c|}{ Jales de la Unidad Minera del sur de México } & \multicolumn{2}{|c|}{ Jales de la Unidad Minera del centro de México } \\
\hline & grises inalterados & café oxidados & grises inalterados & café oxidados \\
\hline Cuarzo $\left(\mathrm{SiO}_{2}\right)$ & $\mathrm{X}$ & $\mathrm{X}$ & $\mathrm{X}$ & $\mathrm{X}$ \\
\hline Calcita $\left(\mathrm{CaCO}_{3}\right)$ & $\mathrm{X}$ & & $\mathrm{X}$ & $\mathrm{X}$ \\
\hline Feldespatos- $\mathrm{K}\left(\mathrm{KAlSi}_{3} \mathrm{O}_{8}\right)$ & $\mathrm{X}$ & $\mathrm{X}$ & $\mathrm{X}$ & $\mathrm{X}$ \\
\hline Wollastonita $\left(\mathrm{CaSiO}_{3}\right)$ & & & $\mathrm{X}$ & $\mathrm{X}$ \\
\hline Pirita $\left(\mathrm{FeS}_{2}\right)$ & $\mathrm{X}$ & & $\mathrm{X}$ & \\
\hline Esfalerita $(\mathrm{ZnS})$ & $\mathrm{X}$ & & $\mathrm{X}$ & \\
\hline Galena $(\mathrm{PbS})$ & $\mathrm{X}$ & & $\mathrm{X}$ & \\
\hline Calcopirita $\left(\mathrm{FeCuS}_{2}\right)$ & & & $\mathrm{X}$ & \\
\hline Yeso $\left(\mathrm{CaSO}_{4} \cdot 2 \mathrm{H}_{2} \mathrm{O}\right)$ & & $\mathrm{X}$ & & $\mathrm{X}$ \\
\hline Goetita $(\mathrm{FeOOH})$ & $\mathrm{X}$ & $\mathrm{X}$ & $\mathrm{X}$ & $\mathrm{X}$ \\
\hline Hematita $\left(\mathrm{Fe}_{2} \mathrm{O}_{3}\right)$ & & & & $\mathrm{X}$ \\
\hline Jarosita $\left(\mathrm{KFe}_{3}\left(\mathrm{SO}_{4}\right)_{2}(\mathrm{OH})_{6}\right)$ & & $\mathrm{X}$ & & $\mathrm{X}$ \\
\hline Caolinita $\left(\mathrm{Al}_{2} \mathrm{Si}_{2} \mathrm{O}_{5}(\mathrm{OH})_{4}\right)$ & & $\mathrm{X}$ & & $\mathrm{X}$ \\
\hline
\end{tabular}


En los jales oxidados de las dos Unidades Mineras predominan los minerales secundarios como yeso $\left(\mathrm{CaSO}_{4} \cdot 2 \mathrm{H}_{2} \mathrm{O}\right)$, jarosita $\left(\mathrm{KFe}_{3}\left[\mathrm{SO}_{4}\right]_{2}(\mathrm{OH})_{6}\right)$, goetita $(\mathrm{FeOOH})$, hematita $\left(\mathrm{Fe}_{2} \mathrm{O}_{3}\right)$ y caolinita $\left(\mathrm{Al}_{2} \mathrm{Si}_{2} \mathrm{O}_{5}(\mathrm{OH})_{4}\right)$, que son los minerales más comunes que caracterizan a los jales oxidados (McGregor et al., 1998; Johnson et al., 2000; Romero et al., 2007). Los minerales secundarios de Fe son los responsables de la coloración café en los jales oxidados de los dos sitios de estudio. Es importante destacar que en los jales oxidados del centro de México se identificaron calcita $\left(\mathrm{CaCO}_{3}\right)$ y wollastonita $\left(\mathrm{SiO}_{3}\right)$, lo que indica que no se agotaron durante los procesos de oxidación-neutralización que ocurrieron en estos jales.

4.2. Concentración total de elementos potencialmente tóxicos (EPT)

Los jales estudiados de las dos unidades mineras seleccionadas tienen concentraciones totales relativamente altas de algunos de los EPT regulados en la Norma Oficial Mexicana de jales NOM-141 (arsénico, cadmio, plomo) y de los elementos propios de los yacimientos minerales de los cuales provienen los jales estudiados (cobre, zinc y hierro). Sin embargo, las concentraciones totales de los otros EPT (bario, cromo, mercurio, plata, plomo, selenio berilio, talio, níquel y vanadio) son relativamente bajas e inferiores a las concentraciones que podrían representar un riesgo para el ambiente y la salud de acuerdo a las Normas Ambientales Mexicanas (SEMARNAT, 2007).

Los jales de la Unidad Minera del sur de México se caracterizan por tener las siguientes concentraciones totales de EPT: arsénico $=140-3627 \mathrm{mg} \cdot \mathrm{kg}^{-1}$, cadmio $=$ $0.5-338 \mathrm{mg} \cdot \mathrm{kg}^{-1}$, plomo $=148-1931 \mathrm{mg} \cdot \mathrm{kg}^{-1}$, cobre $=$ $0.002-0.03 \%$, zinc $=0.125-3.11 \%$ y hierro $=6.7-35.7$ $\%$. Así mismo, en los jales de la Unidad Minera ubicada en el centro de México se determinaron las siguientes concentraciones totales: arsénico $=160-643 \mathrm{mg} \mathrm{kg}^{-1}$, cadmio $=25-434 \mathrm{mg} \cdot \mathrm{kg}^{-1}$, plomo $=300-10900 \mathrm{mg} \cdot \mathrm{kg}^{-1}$, cobre $=0.02-1.55 \%$, zinc $=0.021-3.86 \%$ y hierro $=$ $2.4-5.16 \%$. Estos resultados indican que los jales de la Unidad Minera del sur de México se caracterizan por tener las mayores concentraciones de arsénico y hierro; mientras que los jales del centro de México tienen las mayores concentraciones de cadmio, plomo, cobre y zinc (Tabla 2, Figura 2).

Las concentraciones totales de EPT en los jales de estudio son congruentes con la composición mineralógica reportada en los yacimientos minerales de los que provienen. Aunque en los yacimientos minerales de los dos sitios estudiados no se reportan minerales de cadmio -y en los jales estudiados no se identificaron minerales de cadmio- su presencia puede estar relacionada con la esfalerita, ya que impurezas de cadmio en estos sulfuros metálicos ha sido reportada en estudios anteriores (Romero et al., 2007).

Tabla 2 Concentración total de elementos potencialmente tóxicos (EPT) en las muestras de jales de las Unidades Mineras localizadas en sur y centro de México

\begin{tabular}{lccccc}
\hline \multirow{2}{*}{ Parámetros } & \multicolumn{2}{c}{ Jales del sur de México } & \multicolumn{2}{c}{ Jales del centro de México } & Límite que puede representar riesgo* \\
\cline { 2 - 5 } & mínimo & máximo & mínimo & máximo & 22 \\
\hline $\mathrm{As}\left(\mathrm{mg} \cdot \mathrm{kg}^{-1}\right)$ & 140 & 3627 & 160 & 643 & 5400 \\
$\mathrm{Ba}\left(\mathrm{mg} \cdot \mathrm{kg}^{-1}\right)$ & 0.2 & 43 & 7.7 & 29 & 37 \\
$\mathrm{Cd}\left(\mathrm{mg} \cdot \mathrm{kg}^{-1}\right)$ & 0.5 & 338 & 25 & 434 & 280 \\
$\mathrm{Cr}\left(\mathrm{mg} \cdot \mathrm{kg}^{-1}\right)$ & 9.3 & 46 & $<5.0$ & 16 & 23 \\
$\mathrm{Hg}\left(\mathrm{mg} \cdot \mathrm{kg}^{-1}\right)$ & $<5.0$ & $<5.0$ & $<5.0$ & $<5.0$ & 390 \\
$\mathrm{Ag}\left(\mathrm{mg} \cdot \mathrm{kg}^{-1}\right)$ & 4.8 & 48.4 & $<4.0$ & 109 & 400 \\
$\mathrm{~Pb}\left(\mathrm{mg} \cdot \mathrm{kg}^{-1}\right)$ & 148 & 1931 & 300 & 10900 & 390 \\
$\mathrm{Se}\left(\mathrm{mg} \cdot \mathrm{kg}^{-1}\right)$ & $<4.5$ & 16 & $<4.5$ & 68 & 150 \\
$\mathrm{Be}\left(\mathrm{mg} \cdot \mathrm{kg}^{-1}\right)$ & $<6.0$ & 8.9 & $<6.0$ & $<6.0$ & 1600 \\
$\mathrm{Ni}\left(\mathrm{mg} \cdot \mathrm{kg}^{-1}\right)$ & 10 & 29 & $<4.2$ & 8.8 & 5.2 \\
$\mathrm{Tl}\left(\mathrm{mg} \cdot \mathrm{kg}^{-1}\right)$ & $<1.5$ & $<1.5$ & $<1.5$ & $<1.5$ & 78 \\
$\mathrm{~V}\left(\mathrm{mg} \cdot \mathrm{kg}^{-1}\right)$ & 10 & 40 & 7.2 & 30 & $\mathrm{NR}$ \\
$\mathrm{Cu}(\%)$ & 0.002 & 0.031 & 0.02 & 1.553 & $\mathrm{NR}$ \\
$\mathrm{Zn}(\%)$ & 3.109 & 0.021 & 3.86 & $\mathrm{NR}$ \\
$\mathrm{Fe}(\%)$ & 35.71 & 2.36 & 5.16 & \\
\hline
\end{tabular}

(*) Concentraciones totales de EPT (en suelos) superiores a estos límites puede representar un riesgo para el ambiente y salud humana de acuerdo a la Normatividad Ambiental Mexicana (SEMARNAT, 2007)

$\mathrm{NR}=$ No reportado 

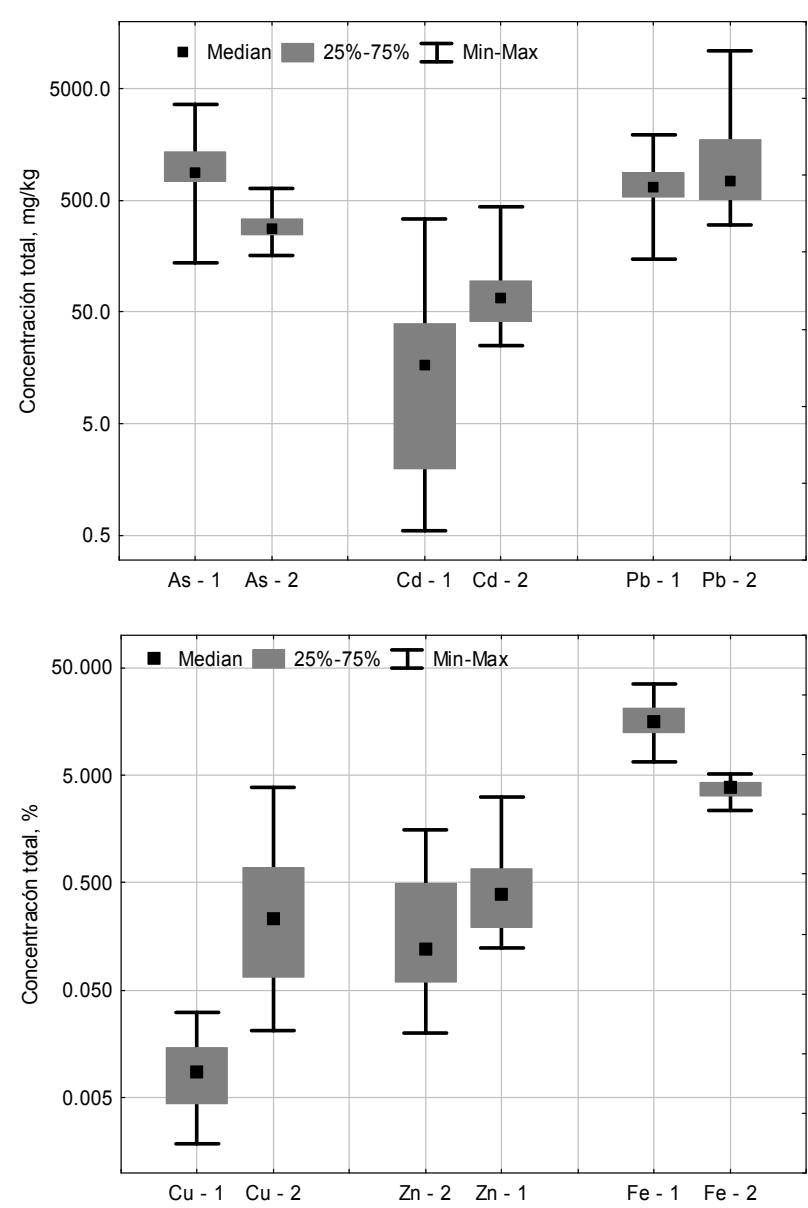

Figura 2 Comportamiento de las concentraciones totales de los elementos potencialmente tóxicos (arsénico "As", cadmio "Cd", plomo " $\mathrm{Pb}$ ", cobre "Cu", zinc "Zn" y hierro "Fe") en los jales de la Unidad Minera del sur de México (As-1, Cd-1, Pb-1, Cu-1, Zn-1 y Fe-1) y de la Unidad Minera del centro de México (As-2, Cd-2, Pb-2, Cu-2, Zn-2 y Fe-2).

\subsection{Capacidad de generación de drenaje ácido}

En los jales del sur de México se determinaron valores de $\mathrm{pH}$ entre 2.4 y 7.8 (Figura 3a). En los jales grises -que no presentan signos visibles de alteración- los valores de $\mathrm{pH}$ son prácticamente neutros $(\mathrm{pH}=7.2-7.7)$; mientras que en los jales oxidados de color café los valores de $\mathrm{pH}$ varían desde ácidos $(\mathrm{pH}=2.4)$ hasta prácticamente neutros $(\mathrm{pH}=7.8)$. En los jales del centro de México, se determinaron valores de $\mathrm{pH}$ prácticamente neutros y ligeramente básicos $(\mathrm{pH}=6.5$ y 8.2$)$ tanto en los jales grises e inalterados como en los jales oxidados (Figura $3 b$ ).

En el caso de los jales oxidados -donde ya ocurrieron los procesos geoquímicos que determinan la generación potencial de drenaje ácido- la determinación del pH es suficiente para valorar su potencial para la generación de drenaje ácido, por lo que se puede concluir que en los jales oxidados del sur de México actualmente coexisten jales que son generadores de drenaje ácido y jales que no lo son. En el caso de los jales oxidados del centro de México, los valores de $\mathrm{pH}$-prácticamente neutros y ligeramente básicos- indican que éstos no son generadores potenciales de drenaje ácido.

Sin embargo, la determinación de los valores de $\mathrm{pH}$ en jales inalterados no es suficiente para valorar su potencial de generación de drenaje ácido, ya que en estos jales no han tenido lugar los fenómenos geoquímicos (oxidaciónneutralización) que determinan la generación potencial de drenaje ácido; por lo que se realizaron pruebas de balance ácido-base (BAB) para pronosticar si serán o no generadores de drenaje ácido en el futuro, cuando se oxiden por la acción de los agentes del intemperismo (agua y aire).

La prueba de BAB se realizó en muestras de jales inalterados de color gris. Los resultados de esta prueba permiten pronosticar que los jales del sur de México serán generadores de drenaje ácido -en el futuro cuando se oxiden- ya que el potencial de neutralización "PN" (media $=121 \mathrm{~kg} \mathrm{CaCO} /$ /tonelada de jal) es inferior al potencial de acidez "PA" (media $=206 \mathrm{~kg} \mathrm{CaCO}_{3}$ /tonelada de jal) (Figura 3c). Con excepción de una muestra, la relación PN/PA varía entre 0.1 y 0.9 (Figura 3d), lo que, de acuerdo a criterios establecidos en la Normatividad Ambiental de México, permite clasificarlos como peligrosos por su capacidad potencial de generación de drenaje ácido. En la Normatividad Ambiental de México los jales se clasifican como generadores potenciales de acidez cuando la relación PN/PA $<1.2$ (SEMARNAT, 2004).

Sin embargo, los jales grises e inalterados del centro de México se clasifican como no generadores potenciales de drenaje ácido (Figura 3c) ya que el potencial de neutralización "PN" (media $=414 \mathrm{~kg} \mathrm{CaCO}_{3} /$ tonelada de jal) es superior al potencial de acidez "PA" (media $=71$ $\mathrm{kg} \mathrm{CaCO}_{3}$ /tonelada de jal). Así mismo, la relación PN/PA varía entre 3.5 y 9.3 (Figura $3 \mathrm{~d}$ ), por lo que de acuerdo a los criterios establecidos en la Normatividad Ambiental de México, estos jales no serán generadores de drenaje ácido en el futuro cuando se oxiden.

4.4. Concentración geodisponible de elementos potencialmente tóxicos (EPT)

En lo jales neutros y ligeramente básicos de la Unidad Minera del centro de México no se favorece la disolución de los minerales que contienen EPT, lo que explica que en sus lixiviados acuosos, la concentración geodisponible de estos elementos es inferior al límite de detección (LD) de la técnica de análisis $\left(\mathrm{LD}_{\text {arsénico }}=0.047 \mathrm{mgL}^{-1}, \mathrm{LD}_{\text {cadmio }}\right.$ $=0.03 \mathrm{mgL}^{-1}, \mathrm{LD}_{\text {cobre }}=0.044 \mathrm{mgL}^{-1}, \mathrm{LD}_{\text {plomo }}=0.06 \mathrm{mgL}^{-1}$, $\left.\mathrm{LD}_{\text {zinc }}=0.057 \mathrm{mgL}^{-1}\right)$. En los jales grises e inalterados estas bajas concentraciones geodisponibles se deben a que los EPT están contenidos en los sulfuros metálicos (Tabla 1), que son químicamente estables bajo las condiciones actuales.

Por otro lado, en el medio neutro y ligeramente básico de los jales oxidados se favorece la precipitación de oxihidróxidos de $\mathrm{Fe}$, los cuales juegan un papel significativo 

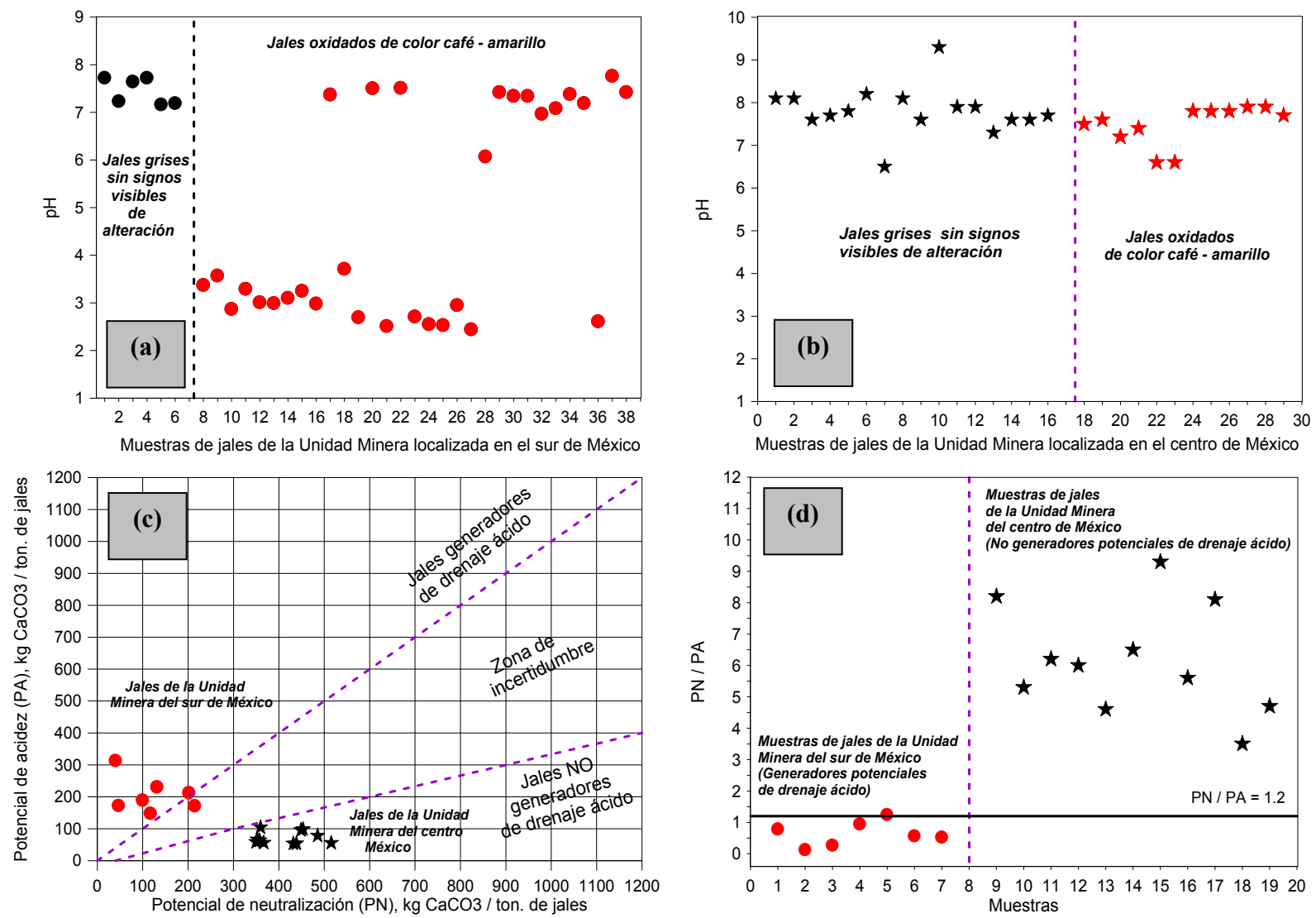

Nota: En la Normatividad Ambiental de México los jales se clasifican como generadores potenciales de acidez cuando la relación PN/PA < 1.2 (SEMARNAT, 2004)

Figura 3 Valores de $\mathrm{pH}$ y de los potenciales de neutralización y de acidez: (a) Valores de $\mathrm{pH}$ en las muestras de jales inalterados y oxidados de la Unidad Minera del sur de México. (b) Valores de $\mathrm{pH}$ en las muestras de jales inalterados y oxidados de la Unidad Minera del centro de México. (c) Valores del potencial de acidez (PA) vs. el potencial de neutralización (PN) de las muestras de jales estudiados. (d) Relación del potencial de acidez (PA) sobre el potencial de neutralización (PN) en las muestras de jales estudiados.

en los procesos de retención de arsénico y metales pesados debido a fenómenos de sorción (Goldberg, 1986; Fuller et al., 1993; Bowell, 1994; Lin, 1997; Foster et al., 1998; McGregor et al., 1998, Romero et al., 2007). Los análisis por Microscopía Electrónica de Barrido acoplada con espectrometría de Rayos X por energía dispersa (MEB-EDS) permitieron identificar que, en los jales oxidados del centro de México, el plomo, el zinc y el cobre están asociados a partículas donde el componente predominante es el Fe (Figura 4a), lo que puede interpretarse como la retención de estos metales pesados en las superficies de oxihidróxidos de $\mathrm{Fe}$-que fueron debidamente identificados por DRXdebido a procesos de sorción, lo que explicaría la baja movilidad de estos EPT.

En los jales de la Unidad Minera del sur de México se determinaron concentraciones geodisponibles de arsénico $\left(<0.047-0.4 \mathrm{mgL}^{-1}\right)$, cadmio $\left(<0.03-0.5 \mathrm{mgL}^{-1}\right)$, plomo $\left(<0.06-0.2 \mathrm{mgL}^{-1}\right)$, zinc $\left(<0.057-55.2 \mathrm{mgL}^{-1}\right)$, hierro $\left(<0.264-28.31 \mathrm{mgL}^{-1}\right)$ y sulfatos $\left(80-4524 \mathrm{mgL}^{-1}\right) . \mathrm{La}$ concentración geodisponible de cobre fue menor al límite de detección $(\mathrm{LD})$ de la técnica de análisis $\left(\mathrm{LD}_{\text {cobre }}=0.044\right.$ $\left.\mathrm{mgL}^{-1}\right)$.
En los lixiviados acuosos de los jales oxidados y ácidos se determinaron los valores máximos de las concentraciones geodisponibles de EPT, debido a que en el medio ácido se favorece la disolución de minerales que contienen a estos EPT. En cambio, en los lixiviados acuosos de los jales oxidados y jales grises inalterados, que tienen valores de $\mathrm{pH}$ entre 7.0 y 7.8, se determinaron las más bajas concentraciones geodisponibles de los elementos tóxicos. En los jales grises e inalterados estas bajas concentraciones geodisponibles se deben a que los elementos de interés están contenidos en los sulfuros metálicos (Tabla 1), los cuales son estables químicamente, mientras no se oxiden.

Los análisis por MEB-EDS permitieron identificar que en los jales oxidados neutros del sur de México, el arsénico, plomo, zinc y cobre están asociados a partículas donde el componente predominante es el Fe (Figura 4b), lo que puede interpretarse como la retención de estos EPT en las superficies de oxihidróxidos de $\mathrm{Fe}$-que fueron debidamente identificados por DRX- debido a procesos de sorción, lo que explicaría la baja movilidad de estos EPT. La presencia de Al, Ca y Si en estas partículas se debe a la presencia de cuarzo y feldespatos en la matriz de los jales 

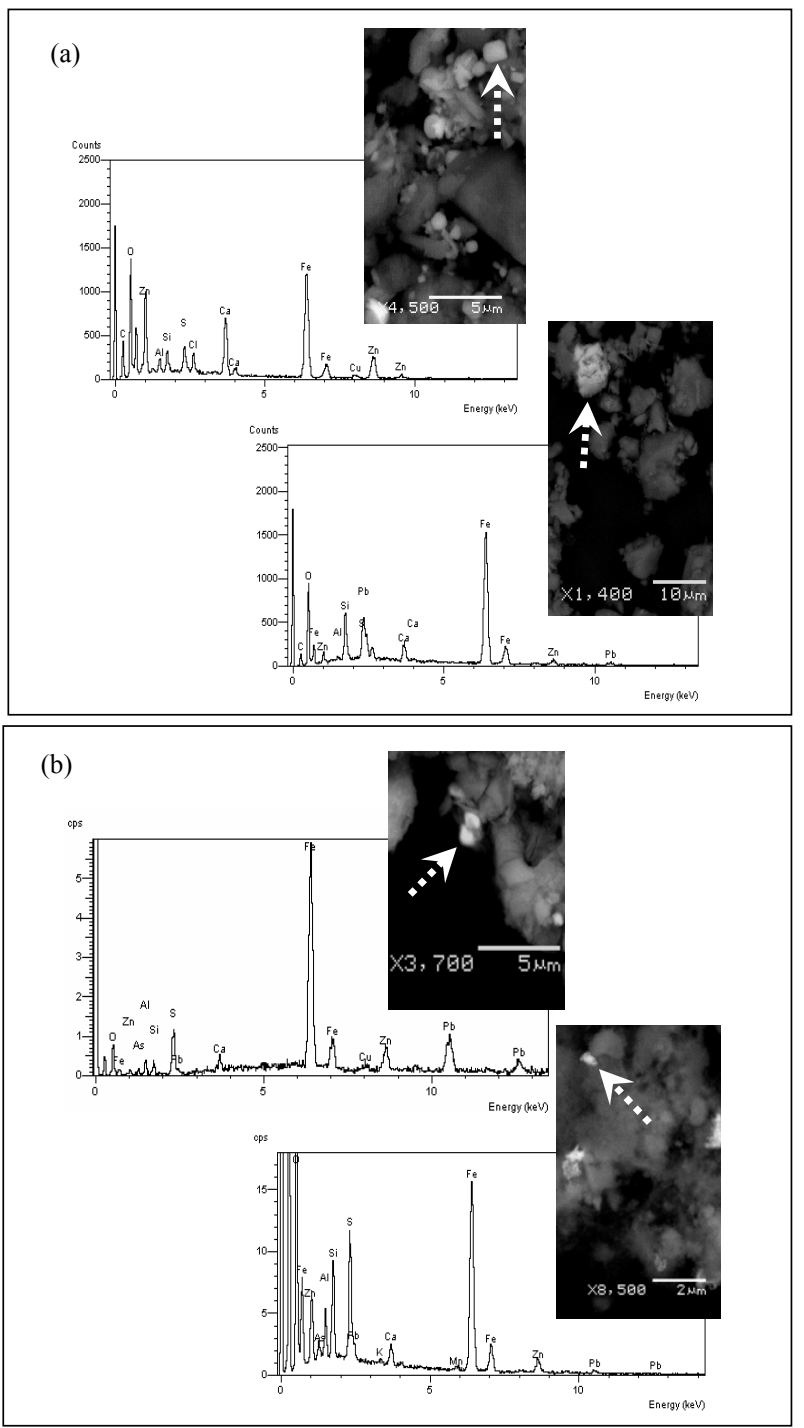

Figura 4 Microfotografías y análisis químico puntual (MEB-EDS) de partículas de oxihidróxidos de hierro en jales de la Unidad Minera del centro de México (a) y del sur de México (b).

de estudio.

Aunque las concentraciones geodisponibles de los EPT en los jales de la Unidad Minera del sur de México sean inferiores a los límites indicados en la NOM 141, su dispersión podría representar un peligro para el entorno. La dispersión de estos lixiviados ácidos con arsénico y metales pesados disueltos es posible en esta zona de estudio debido a la condiciones climáticas. Con el fin de minimizar el riesgo ambiental que representan estos lixiviados ácidos, se están realizando investigaciones que permitan identificar las alternativas para su manejo y control. Actualmente se ha logrado determinar que las lutitas calcáreas de la Formación Mexcala, que se distribuyen ampliamente en la zona de estudio, tienen un alto potencial de neutralización y pueden retener eficientemente arsénico y metales pesados (Romero et al. 2007b), por lo que la construcción de barreras geoquímicas permeables puede ser utilizada para el manejo y control del drenaje ácido de los jales estudiados en este trabajo.

\section{Conclusiones}

En los dos sitios estudiados se identificaron jales inalterados de color gris y jales oxidados de color café. Estos jales se caracterizan por tener concentraciones totales altas de elementos potencialmente tóxicos (EPT) como arsénico, cadmio, plomo, cobre, zinc y hierro.

Los valores de $\mathrm{pH}$ neutros y ligeramente básicos en los jales oxidados del centro de México permiten concluir que éstos no son generadores de acidez. Sin embargo, en los jales oxidados del sur de México, los valores de $\mathrm{pH}$ indican que en este sitio coexisten jales que actualmente son generadores de acidez (valores de $\mathrm{pH}$ ácidos) y jales que no lo son (valores de $\mathrm{pH}$ nuetros y ligeramente básicos). Por otro lado, los resultados de las pruebas de balance ácido-base permiten pronosticar que los jales inalterados y grises del sur de México serán generadores de acidez - en el futuro cuando se oxiden-, ya que su potencial de neutralización es menor que su potencial de acidez. Sin embargo, el potencial de neutralización de los jales inalterados del centro de México es mucho mayor que el potencial de acidez, lo que indica que estos jales no serán generadores de acidez, ya que la cantidad de minerales neutralizadores es suficiente para neutralizar la acidez que generen estos jales -en el futuro- cuando se oxiden.

Los lixiviados ácidos de los jales oxidados del sur de México se caracterizan por contener EPT geodisponibles en concentraciones inferiores a los límites señalados en la NOM-141, mientras que en los lixiviados neutros, de los dos sitios estudiados, no se detectó la presencia de EPT geodisponibles. Estos resultados indican la baja movilidad de estos contaminantes, la cual se puede explicar debido a que éstos están asociados a fases sólidas estables. En el caso de los jales grises inalterados, los EPT están asociados a sulfuros metálicos y en el caso de los jales oxidados, a partículas de oxihidróxidos de hierro debido, probablemente, a procesos de sorción.

\section{Agradecimientos}

Este estudio fue financiado por el proyecto PAPIIT IN105108. Los autores agradecen a Gerardo Martínez, Hedgar Hernández y Alexandra Lara (Laboratorio de Análisis Físicos y Químicos del Ambiente del Instituto de Geografía, UNAM) por su asistencia en la preparación de muestras y análisis de laboratorio. También se agradece a Teresa Pi Puig (Instituto de Geología, UNAM) por los análisis de difracción de rayos X, y a C. Linares (Instituto de Geofísica) e I. Puente Lee (Facultad de Química, UNAM) por su asistencia en los análisis de Microscopía electrónica de barrido (MEB-EDS) 

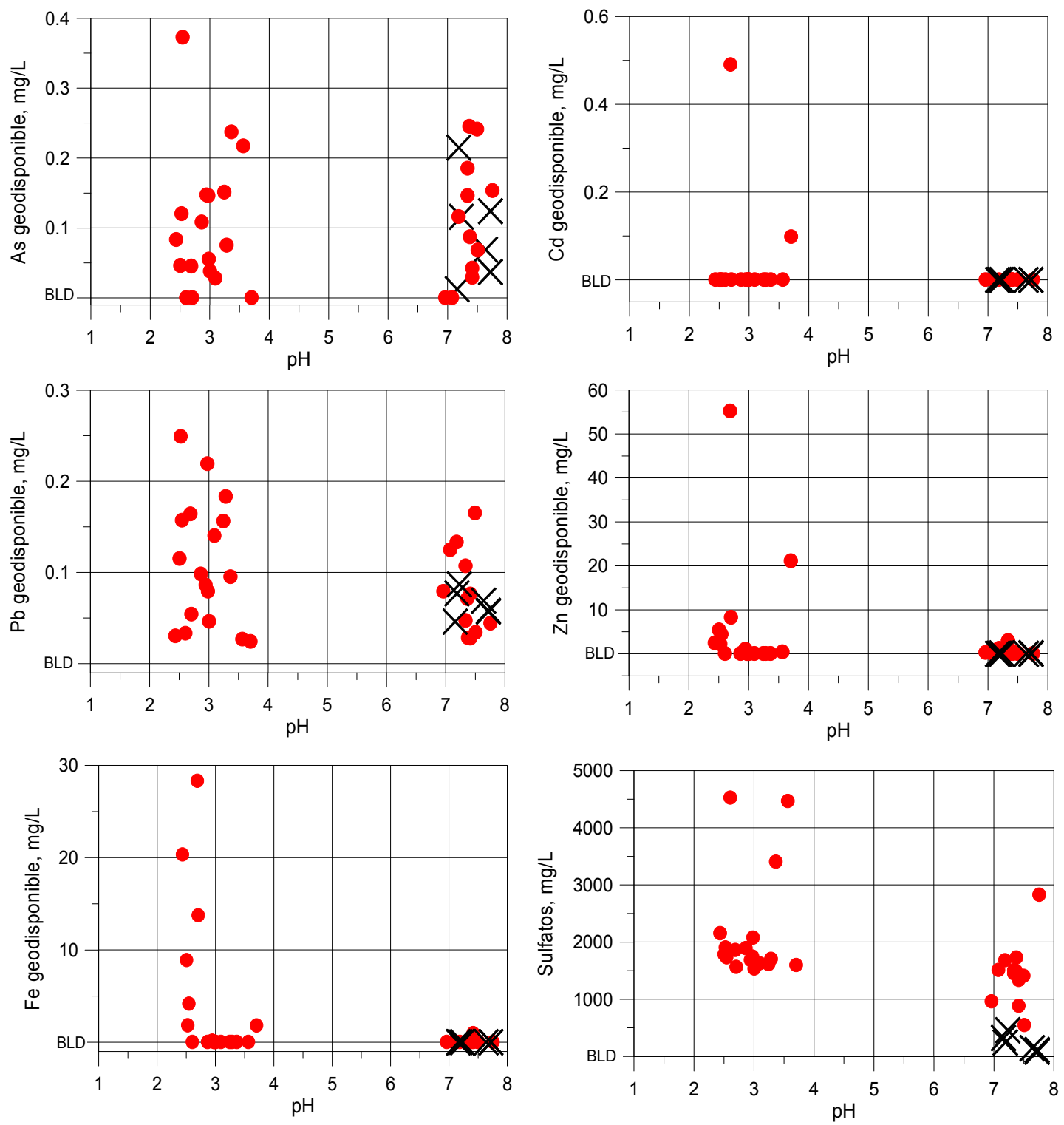

Figura 5 Comportamiento de las concentraciones geodisponibles de los elementos potencialmente tóxicos en función del pH en los lixiviados acuosos de los jales de la Unidad Minera del sur de México: $(\bullet)$ jales cafés oxidados $(X)$ jales grises inalterados.

\section{Bibliografía}

Armienta, M.A., Villaseñor, G., Rodríguez, R., Ongley, L.K., Mango, H., 2001, The role of arsenic-bearing rocks in groundwater pollution at Zimapan Valley, Mexico: Environ. Geol. 40 (4-5), 571-581.

Bain, J.G., Blowes, D.W., Robertson, W.D., Frind, E.O., 2000, Modelling of sulfide oxidation with reactive transport at a mine drainage site: J. Contam. Hydrol. 41 (1-2), 23-47.

Bowell, R.J., 1994, Sorption of arsenic by iron oxides and oxyhydroxides in soils: Appl Geochem 9, 279-286.

Comisión Nacional del Agua (CNA), 2002, Determinación de la disponibilidad de agua en el acuífero Villa de Arista, estado de San Luis Potosí, Gerencia de Aguas Subterráneas, Subgerencia de Evaluación y Modelación Hidrogeológica, México D.F., México.

Consejo de Recursos Minerales (COREMI), 1999, Monografía Geológico Minera del Estado de Guerrero. Consejo de Recursos Minerales, Pachuca, Hidalgo, México.
Goldberg, S., 1986, Chemical modeling of arsenate adsoprtion on aluminium and iron oxide minerals: Soil Sci Soc Am J 50, 1154 -1157

Foster, A.L., Brown, G.E., Tingle, T.N. and Parks, G.A., 1998, Quantitative arsenic speciation in mine tailings using X-ray absorption spectroscopy. American Mineralogist, 83(5-6), 553-568

Fuller, C.C., Davis, J.A., Waychunas, A., 1993, Surface chemistry of ferrihydrite: Part2. Kinetics of arsenate adsorption and coprecipitation: Geochim Cosmochim Acta 57, 2271-2282.

Industrial Minera México Sociedad Anónima (IMMSA), 1973, Yacimientos minerales metálicos del Distrito Minero de Taxco. Reporte interno. Taxco, Guerrero, México.

Instituto Nacional de Estadística, Geografía e Informática (INEGI), 1999, Síntesis Geográfica del Estado de Guerrero. Aguascalientes, México.

Johnson R.H., Blowes, D.W., Robertson, W.D., and Jambor, J.L., 2000, The hydrogeochemistry of the Nickel Rim mine tailings impoundment, Sudbury, Ontario: J. Contam. Hydrol. 41 (1-2), 49-80. 
Jung, M.C., 2001, Heavy metal contamination of soils and waters in and around the Imcheon Au-Ag mine, Korea: Appl. Geochem. 16 (1112), 1369-1375.

Lawrence, R.W., Wang, Y., 1997, Determination of neutralization potential in the prediction of acid rock drainage: Fourth International Conference on acid rock drainage. Vancouver, BC. Canada.

Lin, Z., 1997, Mobilization and retention of heavy metals in mill-tailings from Garpenberg sulfide mines, Sweden: Sci. Total Environ. 198, 13-31.

McGregor, R.G., Blowes, D.W., Jambor, J.L., Robertson, W.D., 1998, The solid-phase controls on the mobility of heavy metals at the Copper Cliff tailings area, Sudbury, Ontario, Canadá: Contam Hydrol 33, 247-271.

Moncur, M.C., Ptacek, C.J., Blowes, D.W., and Jambor J.L., 2004. Release, transport and attenuation of metals from an old tailings impoundment: Appl. Geochem. 20, 639-659

Ramírez, E., 2001, Almacenamiento de residuos mineros en México. Memoria del seminario "Almacenamientos de Residuos Mineros" CNA - SMMS - CMM, México D.F. México.

Romero, F. M., Armienta, M. A., and González-Hernández G., 2007, The solid-phase control on the mobility of potentially toxic elements in an abandoned lead/zinc mine tailings impoundment, Taxco, México: Appl. Geochem. 22: 109-127

Romero, F.M., 2007a. Caracterización de jales y evaluación de su impacto y riesgo en el medio abiótico. Unidad Minera Charcas, San Luis Potosí: México D.F., México, Universidad Nacional Autónoma de México, Instituto de Geología, Reporte Técnico.

Romero, F. M., 2007b. Manejo y control del drenaje ácido en la Unidad Minera de Taxco: Alternativas de tratamiento. Etapa I. Universidad Nacional Autónoma de México, Instituto de Geología, Reporte Técnico.
Secretaría de Medio Ambiente y Recursos Naturales (SEMARNAT), 2004, Norma Oficial Mexicana (NOM-141-SEMARNAT) que establece el procedimiento para caracterizar los jales así como las especificaciones y criterios para la caracterización y preparación del sitio, proyecto, construcción, operación y pos-operación de presas de jales, Diario Oficial de la Federación, 14 enero de 2004. México.

Secretaría de Medio Ambiente y Recursos Naturales (SEMARNAT), 2007, Norma Oficial Mexicana (NOM-147-SEMARNAT/SSA1) que establece los criterios para determinar las concentraciones de remediación de suelos contaminados por arsénico, bario, berilio, cadmio, cromo hexavalente, mercurio, níquel, plata, plomo, selenio, talio y/o vanadio. Diario Oficial de la Federación, 2 de marzo de 2007. México.

United States Environmental Protection Agency (US-EPA), 1994, Method 3051: Microwave assisted acid digestion/sludges, soils: Test Methods for Evaluating Solid Waste, Physical/Chemical Methods, http://www.epa.gov/sw-846/pdfs/9045d.pdf

United States Environmental Protection Agency (US-EPA), 1995, Method 9045C: Solid and waste pH, Test Methods for Evaluating Solid Waste, Physical/Chemical Methods, http://www.epa.gov/ sw-846/pdfs/9045d.pdf

United States Environmental Protection Agency (US-EPA), 1996, Method 6010b: Inductively Coupled Plasma-Atomic Emission Spectrometry, Test Methods for Evaluating Solid Waste, Physical/ Chemical Methods, http://www.epa.gov/sw-846/pdfs/6010c.pdf

Recibido: $15 / 05 / 2008$

Recibido corregido: 23/09/2008

Aceptado: 10/10/2008 